\title{
Concentric Tube Vacuum Chamber for High Magnetic Field, High-Pressure Ionization in a Fourier Transform Ion Cyclotron Resonance Mass Spectrometer
}

\author{
S. A. Hofstadler*, E. Schmidt, Z. Guan, and D. A. Laude, Jr. \\ Department of Chemistry and Biochemistry, The University of Texas at Austin, Austin, Texas, USA
}

\begin{abstract}
A new differential pumping design for external source Fourier transform ion cyclotron resonance mass spectrometry is described. A network of concentric tubes of increasing diameter terminates at a series of conductance limits across which a pressure from atmosphere to low- $10^{-8}$ torr is achieved. This design permits high-pressure sources to be positioned within the solenoidal superconducting magnet less than $20 \mathrm{~cm}$ from the analyzer trapped ion cell. Ionization at high magnetic field offers the advantage of radial ion confinement and consequently delivers enhanced ion current to the trapped ion cell. Ion injection utilizing this vacuum chamber design is simpler than previously reported serial pumping stage designs because elaborate focusing optics to overcome the magnetic mirror effect are unnecessary. Two probe-mounted atmospheric pressure sources are described as evidence of the general applicability of the concentric tube vacuum chamber. An electrospray source that delivers several hundred picoamperes of ion current to the cell yields high-sensitivity spectra of proteins beyond $100 \mathrm{kDa}$. Improved pumping compared with a prototype concentric tube network configuration now permits mass resolution in excess of 20,000 for the $[\mathrm{M}+4 \mathrm{H}]^{4+}$ ion of melittin. The resolution is sufficient to distinguish isotope peaks within a single charge state. A probe-mounted, pulsed-laser ablation source that permits cluster formation in the strong magnetic field is also demonstrated. (J Am Soc Mass Spectrom 1993, 4, 168-176)
\end{abstract}

\begin{abstract}
A $\mathrm{s}$ a consequence of low-pressure detection requirements for Fourier transform ion cyclotron resonance (FTICR) mass spectrometry [1-5], only a limited number of low gas load ionization sources, including laser desorption [6-8] and electron ionization [9], are directly compatible with the analyzer trapped ion cell vacuum chamber. As a solution, several FTICR spectrometers have been constructed in which sample introduction and ionization at high pressure is achieved in a spatially distinct region of the vacuum chamber [ $10-30]$. In each case, ions are formed in a differentially pumped source volume and then transported across one or more conductance limits to the analyzer chamber. The similarity of vacuum chamber design for these external sources can be attributed to geometric constraints imposed by the horizontal, solenoidal superconducting magnets typically used for high-performance FIICR.
\end{abstract}

* Present address: Battelle Pacific Northwest Laboratories, Richland WA 99352 .

Address reprint requests to D. A. Laude, Jr., Department of Chemistry, The University of Texas at Austin, Austin, TX 78712 .
The simplest design for a differentially pumped vacuum chamber is achieved by appending pumps to the chamber at both ends of the magnet; a conductance limit with a 2 to $5 \mathrm{~mm}$ orifice positioned between the two pumps permits a pressure differential between source and analyzer volumes that is on the order of $10^{2}-10^{3}[10]$. With this vacuum chamber arrangement, chemical ionization [11], collision-induced dissociation [12], and introduction of gas chromatographic effluent [13] can be accomplished in an ionization source maintained in the $10^{-5}$-torr region, with ions subsequently transferred across the conductance limit to a $10^{-8}$-torr analyzer region for detection. One of the first examples of this type of two-section chamber was the Nicolet dual trapped ion cell for which the conductance limit also served as a common trap plate for source and analyzer cell F[ICR detection [10]. Other two-section chambers use alternative dual cell designs that promote mass selection or ionization duty cycle advantages [14-18].

If ion sources operating at pressures exceeding $10^{-5}$ torr are to be coupled directly to the low-pressure FTICR detection region, then additional pumping 
stages must be appended to the vacuum chamber. As shown in Figure 1a, the near-universal approach to this multichamber design is a linear series of pumping stages extending away from the cell along the magnetic field axis [19-30]. With as many as five stages of pumping in series, sources operated at greater than atmospheric pressure are possible. Examples of highpressure sources that have been interfaced to FTICR include high-pressure laser ablation [23-25], Cs' secondary ion mass spectrometry [26], fast-atom bombardment [22], and electrospray ionization (ESI) [28-30]. Unfortunately, in an effort to solve the pressure mismatch between source and analyzer, these external source devices introduce new obstacles to effective execution of the FTICR experiment. Specifically, because ions are formed outside the strong magnetic field, it is necessary that appropriate ion optics be used to efficiently transfer ions across the magnetic field gradient. Both electrostatic and quadrupole ion guide assemblies have been successful in transporting detectable ion populations to the cell, although reported estimates for ion transport and trapping efficiencies vary widely.

In an effort to simplify the ion transfer process for external source FTICR, we have undertaken a new approach to vacuum chamber design. As an alternative to the serial design shown in Figure 1a, a network

a

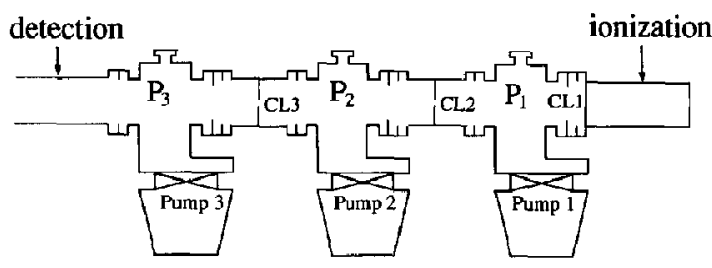

b

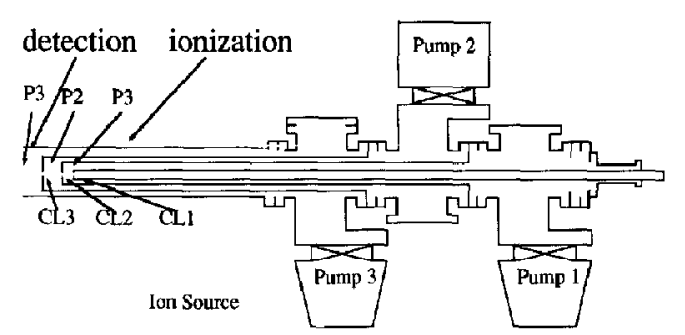

Figure 1. Comparison of (a) linear vacuum chamber and (b) concentric tube vacuum used to provide differential pumping between a high-pressure external source and the low-pressure FTICR trapped ion cell. The advantage of the concentric tube arrangement is that the distance between the source and analyzer volumes can be significantly reduced, which allows ion to be formed at high pressure in the magnetic field. CL1, CL2, and CL3 and P1, P2, and P3 distinguish the conductance limits and pressure, respectively, along the ion beam path. of concentric tubes that are separated at conductance limits in the bore of the superconducting magnet are described. As shown in Figure 1b, the concentric network design spatially consolidates conductance limits so that high-pressure ionization occurs in the magnet bore within a few centimeters of the analyzer cell, which is still maintained at low pressure for FTICR detection. The concentric tube vacuum chamber should offer similar advantages to the dual cell designs in which the source also is positioned in the magnetic field. These advantages include confinement of ions with substantial radial kinetic energies to small $c y$ clotron orbits, unit transfer of ions to the analyzer cell, efficient trapping of ions because axial kinetic energies of a few electron volts per unit charge are compatible with the applied trapping potential, elimination of expensive focusing optics necessary to overcome the magnetic mirror, and reduced spectrometer size. The added benefit is that, whereas the dual cell design can only reach source pressures of $10^{-5}$ torr and still retain optimum conditions for FTICR detection, the concentric tube assembly accommodates sources operating at up to atmospheric pressure.

As a first demonstration of the efficacy of the concentric tube network design, an ESI source for FTICR was developed $[30,31]$. A several hundred-fold increase in ion current delivered to the cell compared with conventional external source ionization with quadrupole injection was evidence of the viability of this new approach to external source vacuum design; however, analyzer cell pressures achieved with these earlier designs were only $10^{-5}$ torr [30] and mid-10 -7 torr [31], in contrast to an analyzer pressure of $2 \times 10^{-8}$ torr obtained with the design presented here. Sufficient mass resolution to separate isotope peaks within a charge state in ESI spectra is now achieved. Another advantage of this latest design is the convenient exchange of probe-mounted high-pressure ion sources; the interface of a high magnetic field, pulsed-laser ablation source for cluster formation [24-26] is also described.

\section{Experimental}

\section{FTICR Spectrometer}

The concentric tube vacuum chamber is housed in the bore of a 3.0-T superconducting magnet with a 6-in. warm bore diameter and 31-in. bore length. A flange-mounted support assembly supports a $5 \mathrm{~cm} \times 5$ $\mathrm{cm} \times 10 \mathrm{~cm}$ elongated cell and provides electrical connections at the cell for excitation, detection, and trap plates. Each trap plate has a $1-\mathrm{cm}$ orifice that is covered by $90 \%$ transmissive stainless steel mesh. The spectrometer is controlled by a Nicolet Analytical Instruments (Madison, WI) 1280-based data station executing Extrel-FTMS (Madison, WI) software version 6.0. Standard Extrel analog electronics include a highpower excitation amplifier capable of $110 \mathrm{Vp}$-p output, preamplifier, and cell controller. 


\section{Concentric Tube Vacuum Chamber}

The primary vacuum chamber is a length of 4-5/8-in.diameter stainless steel tubing with welded ultra-high vacuum (UHV) flanges at both ends, as shown in Figure 2. Double-sided UHV flanges are used to mate concentric tube assemblies to each end of the $4-5 / 8$-in. tube. From the analyzer end of the magnet, a 4-in.diameter tube mated to the primary chamber terminates 17 -in. into the vacuum chamber at an electrically isolated 4-mm conductance limit. This 4-in.-diameter tube is in turn mated to an 8-in. UHV cross. Mounted on the cross are the trapped ion cell flange assembly and a 1200-L/s diffusion pump backed by a $4.7-$ $\mathrm{ft}^{3} / \mathrm{min}$ rotary vane pump.

From the source end of the vacuum chamber, the 4-5/8-in.-diameter tube is mated to a modified 8 -in. UHV cross, which is pumped by a second $1200-\mathrm{L} / \mathrm{s}$ diffusion pump backed by a $4.7-\mathrm{ft}^{3} / \mathrm{min}$ rotary vane pump. Along the concentric tube axis, the source side cross is mated with a 3.5-in.-diameter $\times 29.75$-in.length tube that terminates $2 \mathrm{~cm}$ from the analyzer conductance limit at a $2-\mathrm{mm}$ conductance limit. The 3.5-in.-diameter tube is mated at a double-sided flange with a modified 6-in. UHV cross. Mounted on this cross are cold fingers and an 1100-L/s cryopump that pumps the 3.5-in.-diameter tube. The cryopump is positioned at a $90^{\circ}$ angle with respect to the diffusion pumps to accommodate gate valves mounted on it and the adjacent diffusion pump.

A final 2-3/4-in.-diameter by 20 -in. concentric tube, centered in the 3.5-in. tube, terminates $1 \mathrm{~cm}$ from the conductance limit of the 3.5-in. tube at a copper skimmer cone with a $59^{\circ}$ internal angle and $200-\mu \mathrm{m}$ orifice (with a $330-\mu \mathrm{m}$ orifice used for some preliminary work, the base pressure for ESI reached mid-10 $10^{-7}$ torr). The 2-3/4-in. tube is mated at a double-sided flange with a 2-3/4-in. UHV tee that is connected to a $13-\mathrm{L} / \mathrm{s}$ single-stage rotary vane pump. Along the concentric tube axis, the tee is mated with the probe housing. The probe housing permits the insertion of polished $3 / 4$-in. diameter probes into which high-pressure ionization source and sample introduction systems are mounted. Probes are introduced into the 2-3/4-in. tube to within a few millimeters of the skimmer cone. At this position

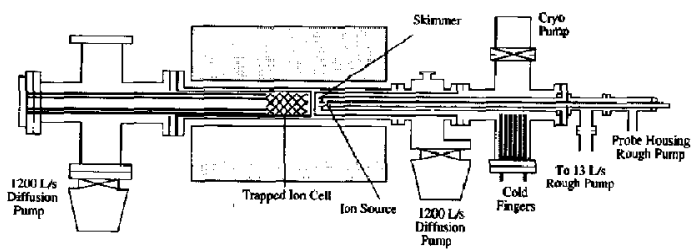

Figure 2 Schematic of the high-pressure, high magnetic field source for FTICR. Atmospheric pressure ionization is accomplished in the source in a $3.0-T$ field a distance of $10 \mathrm{~cm}$ from the analyzer cell, which is maintained below $2 \times 10^{-8}$ torr in the 3.0-T field. in the vacuum chamber ions are formed in a magnetic field estimated at $2 \mathrm{~T}$.

\section{Probe-Mounted Electrospray Source}

As shown in Figure 3a and $b$, the electrospray source is mounted in a hollow 0.75 -in.-diameter by 57 -in. stainless steel tube. It consists of a $100-\mu \mathrm{m}$ i.d. blunt-ended electrospray syringe needle positioned $6 \mathrm{~mm}$ from a $500-\mu \mathrm{m}$ i.d. $\times 20-\mathrm{cm}$ desolvating capillary. A Delrin guide assembly is used to plug the probe and center the syringe needle and desolvating capillary which are adjacent to the skimmer. A spray cavity machined into the Delrin guide assembly between the needle and capillary is the site of ESI. The capillary protrudes several centimeters from the plug to a distance between 1 and $5 \mathrm{~mm}$ from the skimmer. When the ESI probe is inserted into the concentric tube vacuum chamber, a distance of $29 \mathrm{~cm}$ separates the ionization cavity from the cell. Sample is delivered to the syringe needle through Teflon tubing that is attached to an ISCO model SFC-500 microflow syringe pump. Electrical connections are made through the probe housing between the syringe needle and high-voltage power supply and between the desolvating capillary and a

$\mathbf{a}$

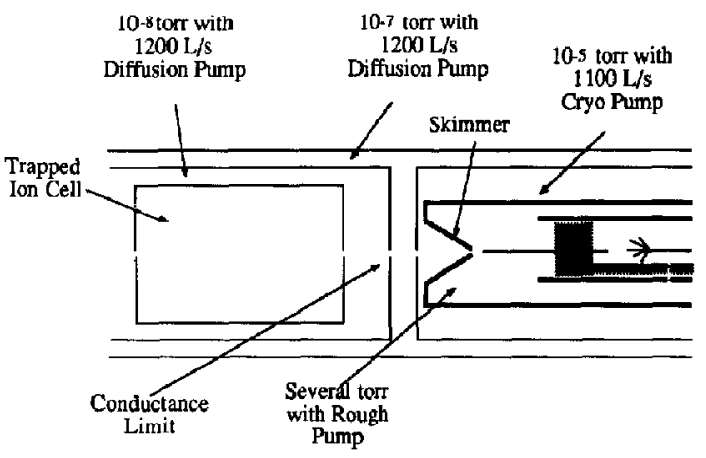

b

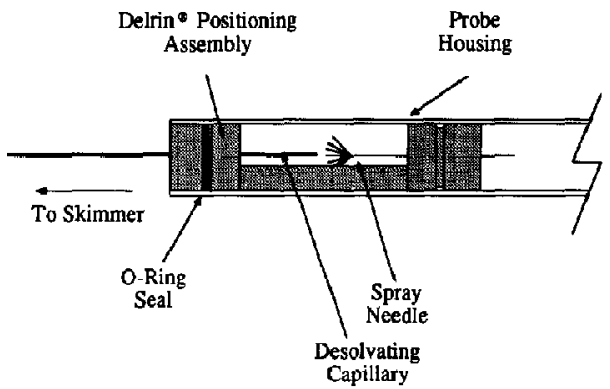

Figure 3. Expanded views of the probe-mounted electrospray source assembly with (a) the probe inserted into the concentric tube assembly and (b) the electrospray source mounted in the probe housing. 
second power supply that resistively heats the capillary.

When operating under normal electrospray conditions with low microliter-per-minute flow rates for methanol/water/acetic acid solutions, a pressure drop from atmosphere in the spray cavity to less than $2 \times$ $10^{-8}$ torr in the analyzer cell is achieved. Concentric vacuum chamber pressures are measured at 2-8 torr in the high-pressure roughing stage, mid-10 $10^{-5}$ torr in the cryopump stage, and $2 \times 10^{-7}$ torr in the first diffusion-pumped stage.

Optimized conditions for efficient formation and trapping of electrospray ions include application of a $3.7-\mathrm{kV}$ electrospray voltage at the syringe needle, a $330-\mathrm{V}$ potential at the desolvating capillary that is maintained at $150{ }^{\circ} \mathrm{C}$, and a variable potential of between 0 and $15 \mathrm{~V}$ at the skimmer. Retarding grid measurements of electrospray ions delivered to the cell indicate that both skimmer potential and capillaryskimmer distance have large effects in determining kinetic energies, which can vary from less than $1 \mathrm{eV}$ to several hundred electron volts. Typically, kinetic energies are controlled at between 0 and $10 \mathrm{eV}$ and matched with trap potentials of $0-10 \mathrm{~V}$ applied to trap plates [31].

The FTICR parameters for low resolution ESI spectra included swept excitation using a $2.66-\mathrm{MHz}$ bandwidth and $1000-\mathrm{Hz} / \mu$ s sweep rate, and detection at a trap potential reduced to $1.0 \mathrm{~V}$. With this interface, sensitivity was significantly improved; for example, a single scan of cytochrome $c$ (molecular weight 12,360.1) following a $500-\mathrm{ms}$ accumulation time would yield a spectral signal-to-noise ratio (S/N) in excess of 100:1. The high mass resolution ESI spectrum to be shown was obtained from $64 \mathrm{k}$ data points acquired over a $20-\mathrm{kHz}$ window following broadband swept excitation.

\section{Laser Ablation Cluster Source}

Shown in Figure $4 a$ and $b$ are diagrams of a probemounted laser ablation source designed for compatibility with the concentric tube inlet system. The source volume, mounted at the end of a 0.75 -in.-o.d, $\times 57$-in. stainless steel tube, is constructed from a $3-\mathrm{cm}$ length $\times 0.60$-in. o.d. Delrin plug fitted with O-rings to form a vacuum seal at the probe terminus. The ionization volume machined from the Delrin plug houses a 4.2$\mathrm{mm}$ i.d. $\times 2-\mathrm{cm}$ aluminum cup that serves as the waiting room for laser-desorbed ions. A 1-mm hole was drilled in the front end of the aluminum cylinder along the skimmer axis. The fiber-optic used to transmit the laser light for surface ablation was introduced through a hole drilled in the back of the Delrin plug. The fiber terminated at the sample surface adjacent to the axis of the sampling orifice and skimmer. To produce the gold spectra to be shown, a piece of gold wire was inserted in the path of the laser light.

A pulsed-valve assembly was constructed to pass nitrogen gas along $1 / 4$-in. tubing in the sample probe, a

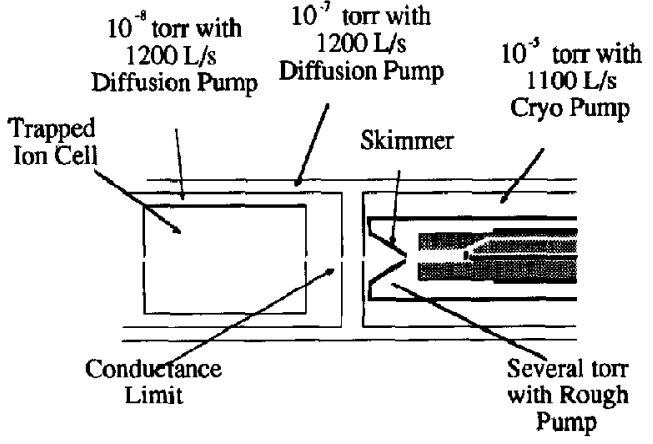

$\mathbf{b}$

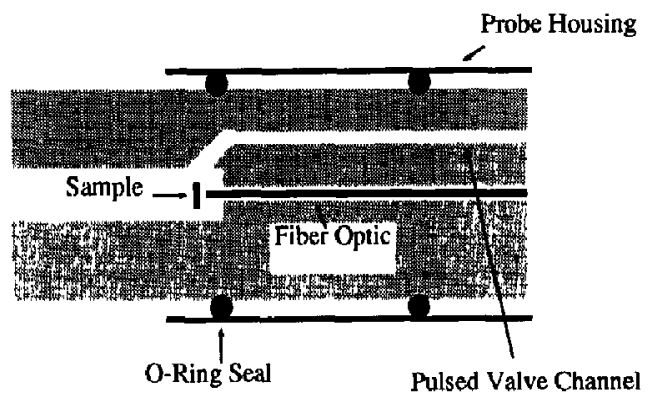

Figure 4. Expanded view of the probe-mounted cluster source assembly with (a) the probe inserted into the concentric tube assembly and (b) the cluster source mounted in the probe housing.

through an opening in the Delrin plug and into the ion volume. Sample pressure in the ion volume prior to laser firing could be varied between atmosphere and $10^{-4}$ torr while maintaining the analyzer pressure at low $10^{-8}$ torr. For pulsed-valve experiments, a background pressure of $10^{-4}$ torr was selected, and pulsed introduction of nitrogen gas increased ion volume pressure to $10^{-2}$ torr. (All pressure measurements for the ion volume were obtained at gauges positioned above the throat of the rough pump, and, thus, actual pressures in the region are higher.)

To perform the laser ablation experiment, a single laser pulse from a Spectra Physics (Mountain View, CA) DCR11 Nd:YAG laser delivered about $30 \mathrm{~mJ}$ of 1064-nm light through the fiber to the sample surface. For an 8-ns pulse and a $0.9-\mathrm{mm}^{2}$ spot size, the laser irradiance in excess of $3 \times 10^{8} \mathrm{~W} / \mathrm{cm}^{2}$ was sufficient to form a plasma. The pulsed valve was triggered to introduce the collision gas to the ionization volume coincident with the laser firing. Clusters were delivered from the ion volume in a weak supersonic expansion (Mach number of about 4) and traversed the network of concentric tube conductance limits to arrive at the cell. In contrast with Smalley's work, gated trapping was unnecessary to acquire ions for FTICR detection, and instead trap plates were maintained at 
constant potential between 1 and $5 \mathrm{~V}$. Under plasma conditions, large numbers of ions traversing the concentric tube network were captured in the cell. High $\mathrm{S} / \mathrm{N}$ broadband FTICR spectra were generated for a 2.66-MHz bandwidth and $16-32 \mathrm{~K}$ data points.

\section{Results and Discussion}

The most recent configuration of the concentric tube external source was designed with the goal of reducing the ultimate pressure achieved at the analyzer cell, improving ion optical alignment at conductance limits along the path to the cell, and permitting rapid change between probe-mounted ionization sources. As is described later, the new system shown in Figure 2 satisfies these design criteria.

\section{Electrospray Operation}

The impetus for constructing the concentric tube external source is that ions generated in a strong magnetic field are radially confined. For example, an ion of $\mathrm{m} / \mathrm{z}$ 1000 with $10 \mathrm{eV}$ of kinetic energy transverse to a 3.0-T field will assume a radial orbit of about $2 \mathrm{~cm}$. Consequently, the entire ion population should be directed toward the detector, in this case the trapped ion cell, with minimal loss. In effect, the magnetic field serves to level the radial kinetic energy of an ion population, a feature that is not realized with electrostatic optics.

Presented in Figure 5 is a profile of electrospray ion current measured in the bore of a 3.0-T superconducting magnet. The current is measured with a Faraday cup of 2-cm diameter at increasing displacement from a 330- $\mu \mathrm{m}$ skimmer cone assembly. The initial current measurement of $360 \mathrm{pA}$ adjacent to the skimmer is maintained over a $30-\mathrm{cm}$ distance before attenuation as field lines diverge on exiting the magnet bore. At a

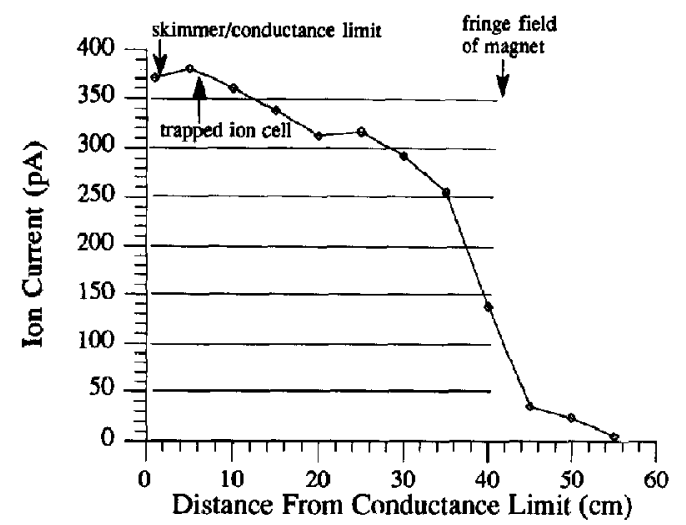

Figure 5. Faraday cup measurement of concentric tube chamber electrospray ion current in the bore of a 3.0-T superconducting magnet. The Faraday cup is displaced over $40 \mathrm{~cm}$ from a position adjacent to the skimmer cone to a region in the fringing magnetic field. Ion current is generated from the $[\mathrm{M}+2 \mathrm{H}]^{2+}$ of gramicidin-S. distance $5 \mathrm{~cm}$ from the skimmer, which corresponds to the position of the trapped ion cell, there is effectively no loss of ion current. The data verify our original premise in designing this type of external source that maximum ion current can be delivered to the trapped ion cell by forming ions at high pressure in a strong magnetic field. Similar results were obtained with the 200- $\mu \mathrm{m}$ skimmer cone.

Because the concentric tube design permits ion formation in the magnetic field, the magnetic mirror effect is circumvented and elaborate focusing optics and high-energy ion translation are unnecessary. Instead, a different set of factors that control the kinetic energy of ions delivered to the cell must be considered. Specifically, the energy of ions at the trapped ion cell is a function of their velocity in the supersonic expansion and the electric field experienced on exiting the skimmer cone. The factor found to be most important in controlling both ion velocity and analyzer pressure, and consequently, FTICR signal intensity, is the distance between the heated capillary and the skimmer cone. Presented in Figure $6 a$ is a profile of trapped ion cell pressure achieved at increasing distance between the skimmer and capillary. Pressure is observed to vary significantly with even small changes in capillary position because sampling occurs at different regions in the supersonic expansion. In general, the pressure minimum in the analyzer cell corresponds to a position of approximately $0.7 x_{\mathrm{m}}$, where $x_{\mathrm{m}}$ is the position of the Mach disk where the expansion terminates.

Presented in Figure $6 \mathrm{~b}$ is the FTICR signal magnitude for several electrospray spectra of the gramicidin-S $[\mathrm{M}+2 \mathrm{H}]^{2+}$ ion acquired at increasing capillary displacement from the skimmer. FTICR performance also is found to depend strongly on the capillary/skimmer distance, with the optimum signal magnitude achieved near the pressure minimum. Thus, to optimize capillary/skimmer position for FTTCR detection, the analyzer pressure is monitored during ESI as the probe is inserted. When the analyzer ion gauge pressure reading is at a minimum, then electrospray kinetic energies will be sufficiently low that FTICR detection will be efficient and spectral quality will be high.

Optimum conditions for trapping electrospray ions were described previously [31]. Briefly, the single requirement to ensure ion accumulation in the cell is that the trap plate potential equal the ion kinetic energy. The kinetic energy can be controlled either by varying the skimmer/capillary distance or the potential applied to the skimmer cone. When conditions are appropriate, ions rapidly accumulate in the cell at a fixed trap potential with cell capacity reached within a few hundred milliscconds.

\section{High-Performance Electrospray/FTICR}

The primary purpose in coupling electrospray to a high-performance mass spectrometer is to realize im- 

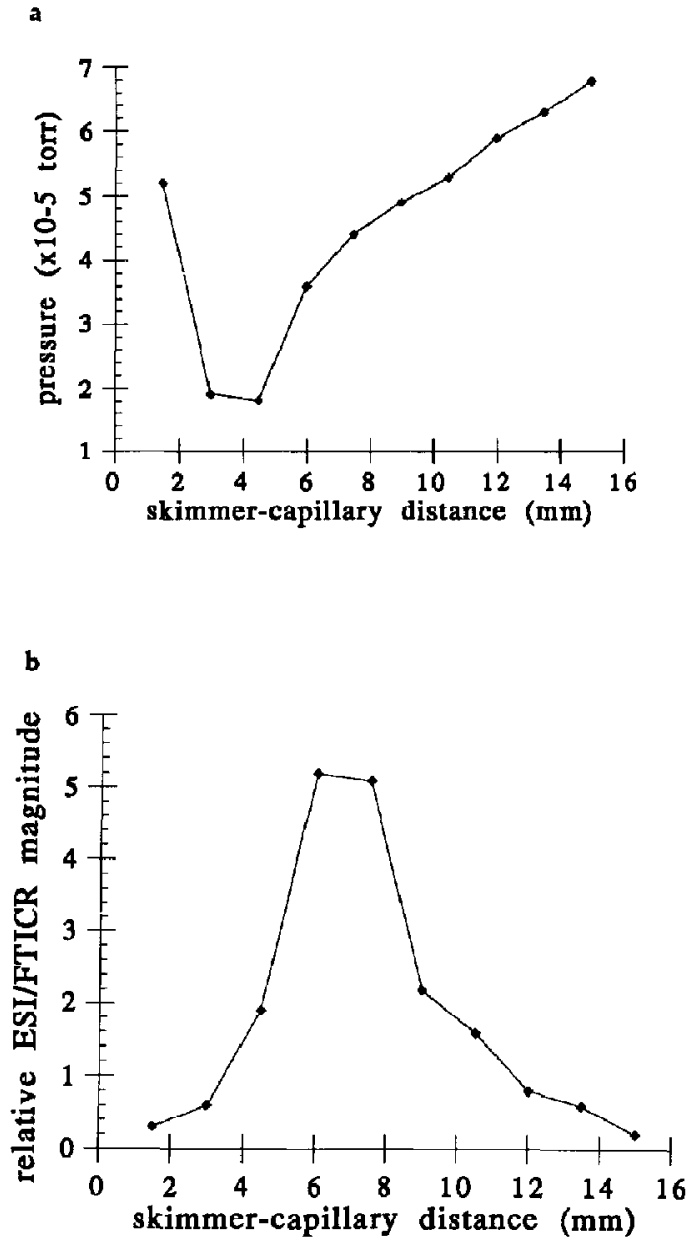

Figure 6. Electrospray FTICR profiles of (a) analyzer cell pressure and (b) FTICR signal magnitude as a function of distance between the desolvating capillary and the skimmer cone. For each profile, the sample is gramicidin- $\mathrm{S}$.

proved high mass-to-charge ratio, mass resolution, and mass accuracy performance compared with quadrupole mass analyzers. Presented in Figure 7 are examples of electrospray mass spectra that cannot be achieved on a quadrupole instrument. Shown in Figure $7 \mathrm{a}$ is a lowresolution spectrum of bovine albumin oblained following a proton transfer reaction. The $[\mathrm{M}+13 \mathrm{H}]^{13+}$ ion at $m / z 5098$ is observed. Presented in Figure $7 \mathrm{~b}$ is a high-mass resulution of the $[\mathrm{M}+4 \mathrm{H}]^{4+}$ ion of melittin. The resolution of 20,000 is sufficient to resolve the individual isotopes within a single charge state. This result can only be achieved by operating the analyzer cell at low pressure and thus requires the use of several stages of differential pumping. The concentric tube network provides an alternative to the external source designs, which must use elaborate injection optics to deliver ions to the trapped ion cell with appropriate kinetic energies and trajectories. McLafferty
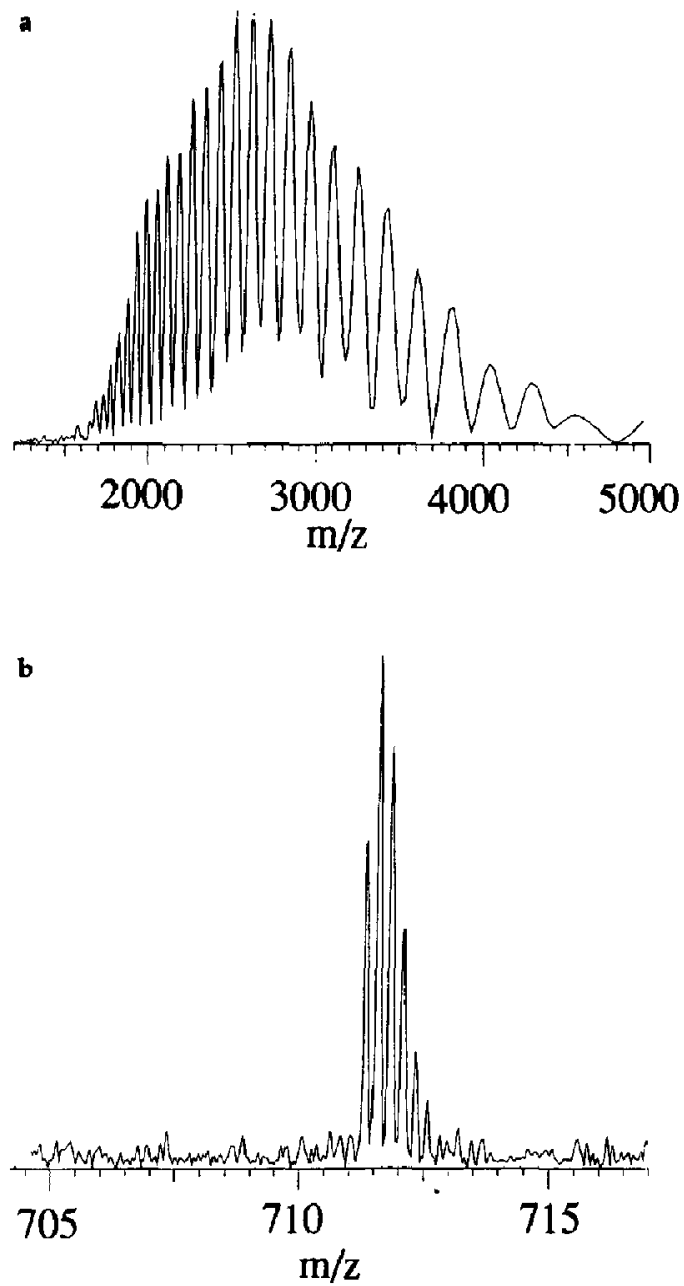

Figure 7. ESI/FTICR spectra of (a) bovine serum albumin following a 10-s deprotonation reaction acquired under broadband conditions to demonstrate mass-to-charge ratio range, and (b) melittin acquired under high-resolution conditions at $2 \times 10^{-8}$ torr to resolve isotopes in the $4+$ charge state.

and co-workers [27-29] have been successful in injecting electrospray-generated ions using such an external source quadrupole assembly. With analyzer pressures in the $10^{-9}$ torr range they have achicved resolving power in excess of 60,000 for cytochrome $c$ ions consuming femtomolar quantities of analyte; however, analysis of larger proteins using this injection scheme has been stymied by relatively low ion current throughput and a relatively broad kinetic energy distribution of ions reaching the trapped ion cell.

\section{Pulsed High-Pressure Cluster Source}

One of the more successful external source instruments designed for FTICR detection of ions formed at highpressure is the laser ablation source/FTICR con- 
structed by Smalley and co-workers [24-26] to study cluster structure and reactivity. Most prominent among the chemical systems investigated are carbon clusters, and principally, $C_{60}$ and $C_{70}$. Smalley et al. have devised two interfaces to couple the high-pressure laser ablation ion source with low-pressure FTKCK detection. For the first approach [24], cluster ions are delivered out of the source by a pulse of inert gas and cooled in a supersonic expansion. The ions are sampled at a right angle to the source and delivered through a series of electrostatic elements that collimate and accelerate ions through the fringing magnetic field and then decelerate and trap the ions for detection. A second, more compact pulsed source and interface [25] is mounted collinear with the magnetic field. Ions are carried from the source in a gas pulse, cooled through a supersonic expansion, and then trapped for detection.

Extending the idea advanced by Smalley of miniaturizing the source and moving it closer to the trapped ion cell, the feasibility of probe mounting a pulsed-laser ablation source for cluster formation was evaluated. The important criteria in constructing the source were that it be probe mounted to mate with the concentric tube pumping system and that ionization occur in the strong magnetic field to eliminate electrostatic ion optics. Because the concentric tube assembly already included a skimmer cone, it was possible to construct a relatively simple sampling volume in which clusters could be generated. The $20-\mathrm{cm}$ length of desolvating capillary used in the probe-mounted electrospray source was unnecessary for the cluster source; thus, the desorption cavity was positioned immediately adjacent to the skimmer cone, which was only $9 \mathrm{~cm}$ from the trapped ion cell. This configuration allows cluster formation to oscur at a magnetic field strength of $3 \mathrm{~T}$. As shown in Figure 4, a target is positioned in the waiting room adjacent to the fiber-optic that delivers the laser pulse. A pulse of gas momentarily increases the pressure in the waiting room by several orders of magnitude and delivers cluster ions across the skimmer to the cell.

Shown in Figure 8 are FTICR spectra derived from a gold target. The spectrum in Figure 8 a results when laser plasma ignition occurs at the gold target, but the pulsed valve is not triggered. A single, intense monatomic ion signal is observed. This lack of clustering mimics results obtained for conventional low-pressure laser ablation of metal targets. The distinction here is that the ions are formed at $10^{-4}$ torr pressure and must traverse three conductance limits to be detected. Identical spectra also are obtained over a range of static pressures extending up to atmospheric pressure. Evidently, for metal targets the plasma ignition conditions that result generate a sufficient number of monatomic ions with appropriate trajectories to arrive at the cell. In contrast, no FTICR signal is detected when organic ion species are thermally desorbed at these high-pressure conditions.

When a pulsed valve is used to create an expansion out of the waiting room and through the skimmer

\section{a}

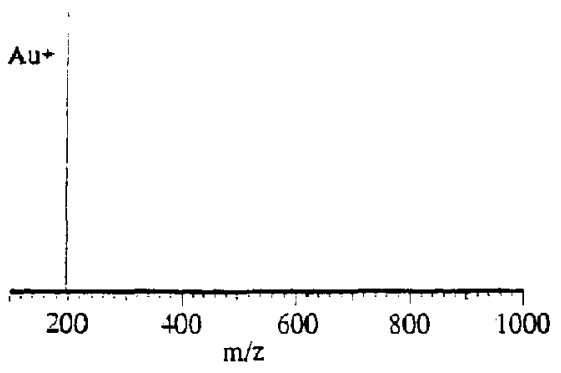

b

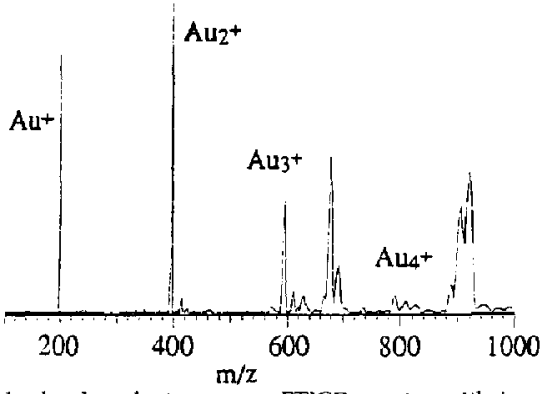

Figure 8. Pulsed-valve cluster source FTICR spectra with ions formed by pulsed-laser desorption at $3 \times 10^{\mathrm{g}} \mathrm{W} / \mathrm{cm}^{2}$ on a gold target with (a) the pulsed-vaive closed and background pressures at $10^{-1}$ torr, and (b) the pulsed-valve is opened to admit nitrogen gas to the waiting room conincident with laser ablation.

cone, cluster ions are observed in the FTICR spectrum. Figure $8 \mathrm{~b}$ is a spectrum acquired under identical conditions to that in Figure 8a, except that a burst of $\mathbf{N}_{2}$ gas was timed to arrive at the waiting room at the appropriate time relative to the laser firing to maximize cluster formation. Gold cluster ions extending to $n=4$ are observed in positive ion mode. Low-resolution peaks result because the spectrum was acquired in broadband mode to observe the entire cluster ensem ble. Additional peaks in Figure $8 \mathrm{~b}$ are due to charge exchange and cation attachment with background neutrals.

The possibility that cluster formation is the result of collisional thermalization rather than cooling in the expansion can be disregarded. If collisional thermalization were the primary factor influencing cluster growth, spectra such as that shown in Figure 8a acquired at pressures from atmosphere to $10^{-4}$ torr would exhibit cluster peaks but do not. Instead, the factor that distinguishes the spectra in Figure 8a and $b$ is the addition of the pulsed $\mathrm{N}_{2}$ gas to generate the latter spectrum. Still to be determined is whether the sensitivity gains realized for the concentric tube electrospray, by exploiting radial confinement, will be realized for the probe-mounted cluster source.

An interesting feature of the procedure used to acquire the cluster spectrum in Figure 8 that distinguishes it from external cluster source spectra is that 
the ions are accumulated in the trapped ion cell without varying the trap plate potential. This is analogous to the electrospray experiment except that no effort was made to match ion kinetic energies and trapping potential. In Smalley's experiment, considerable effort is devoted to timing the gated deceleration and injection of ions into a three-electrode trap that features an additional "screen door" electrode. One explanation for why the concentric tube source does not require this gated trapping procedure follows from a consideration of the differences in charged-particle populations that encounter the cell. In Smalley's original cluster work [24], a pulsed repeller electrode directed a charged-particle population of desired polarity at a right angle to the source onto magnetic field lines. This nonneutral ion population depended on temporal differences in the electric field experienced by ions to be trapped in the cell for detection. Hence, gated trapping was used to enhance the effect. In contrast with this gated trapping experiment, we have shown that a neutral plasma of positive and negative charges creates a shield for low-energy ions to permit the penetration of large trapping electric fields that would otherwise repel the low-energy ions [33]. This plasmashielding phenomenon was suggested as a mechanism to account for the trapping of abundant ion populations formed by laser plasma ignition of metals. Apparently, the mechanism can also be used to account for the trapping of cluster ions from the concentric tube source, because with this interface no distinction is made between positive and negative charged particles in the source or along the grounded flight path to the cell. Consequently, a neutral charged-particle ensemble containing the cluster ions is likely to encounter the trapping electric fields and penetrate. Deshielding effects in the dynamic plasma would be responsible for the subsequent trapping of cluster ions in the cell.

\section{Conclusions}

A new approach to external source FTICR is demonstrated in which ions are formed at high pressure in the bore of the superconducting magnet and traverse a differentially pumped concentric tube network, to arrive at a trapped ion cell that is maintained at low $10^{-8}$ torr. Because ion formation occurs in the high magnetic field, elaborate focusing optics are unnecessary to overcome the magnetic mirror effect. The vacuum chamber is designed to be compatible with a variety of probe-mounted high-pressure ionization sources. Examples presented here include a probemounted electrospray ionization source and a probemounted laser ablation cluster source. Both an isotope-resolved, high-resolution spectrum and an extended mass-to-charge ratio range spectrum of proteins are demonstrated with the electrospray interface. An FIICR spectrum of gold with clusters to $n=4$ is obtained with the pulse, high-pressure cluster source.
A variety of high-pressure ion sources, such as chemical ionization, glow discharge, and thermospray, are currently being evaluated for compatibility with the concentric tube network.

\section{Acknowledgments}

This work is supported by the Welch Foundation, the Arnold and Mabel Beckman Foundation, the Texas Advanced Research Program, the National Institutes of Health, and the National Science Foundation.

\section{References}

1. Wanczek, K. P. Int. J. Mass Spectroth. Ion Processes 1989, 95, 1-38.

2. Wang, M.; Marshall, A. G. Anal. Chem. 1990, 62, 51b-520.

3. Laude, D. A. Jr.; Johlman, C. L.; Brown, R. S.; Weil, A.; Wilkins, C. L. Mass Spectrom. Reo. 1986, 5, 107-119.

4. Marshall, A. G. Acc. Chem. Res. 1985, 18, 316-322.

5. Gross, M. L.; Rempel, D. L. Science 1984, 225, 261-266.

6. Nuwaysir, L. M.; Wilkins, C. L. In Lasers and Mass Spectrometry (Oxford Series on Optical Sciences); Lubman, D. M., Ed.; Oxford University Press: New York, 1989; Chapter 13.

7. Shomo, R. E.; Marshall, A. G.; Weisengerger, C. R. Anal. Chem. 1985, 57, 2740-2744.

8. Jjames, C. F.; Wilkins, C. L. J. Am. Chem. Soc. 1988, 110, 2687-2688.

9. Wilkins, C. L.; Gross, M. L. Anal. Chem. 1981, 53 . $1661 \mathrm{~A}-1666 \mathrm{~A}$.

10. (a) Cody, R. B.; Kinsinger, J. A.; Ghaderi, S.; Amster, I. J.; McLafferty, F. W.; Brown, R. S. Anal. Chim. Acta 1985, 178, 43-66; (b) U.S. Patent Number 4581533.

11. Cody, R. B. Anal. Chem. 1989, 61, 2511-2515.

12. Cody, R. B.; Burnier, R. C.; Cassady. C. J.; Frieser, B. S. Anal. Chem. 1982, 54, 2225-2228.

13. Hogan, J. D.; Laude, D. A. Jr. Anal. Chem. 1989, 61, 2422.

14. Kerley, E.; Russell, D. Anal. Chem. 1989, 61, 53-57.

15. Hanson, C. D.; Kerley, E. L.; Russell, D. H. Anal. Chem. 1989, $61,83-85$.

16. Giancaspro, C; Verdun, F. R.; Muller, J. F. Int. J. Mass Spec. Ion Processes 1986, 72,63 .

17. Wise, M. B. Anal. Chem. 1987, 59, 2289-2293.

18. Hofstadler, S. A.; Laude, D. A. Jr. Anal. Chem. 1991, 63, 2001-2007.

19. Kofel, P.; Alleman, M.; Kellerhals, H.; Wanczek, K. P. Int. J. Mass. Spec. Ion Processes 1989, 87, 237-247.

20. Kofel, P.; Alleman, $M_{i}$; Kellerhals, H.; Wanczek, K. P. Int. J. Mass. Spec. Iun Prucesses 1985, 65, 97-103.

21. Kofel, P.; Alleman, M.; Kellerhals, H.; Wanczek, K. P. Int. J. Mass. Spec. Ion Processes 1986, 72, 53-61.

22. Lebrilla, C. B.; Amster, I. J.; McIver, R. T. Jt. Int. J. Mass SpecIon Processes 1989, 87, R7-R13.

23. Alford, J. M.; Williams, P. E.; Trevor, D. J.; Smalley, R. E. Int. I. Mass. Spec. Ion Processes 1986, 72, 33-51.

24. Smalley, R. E. Anal. Instrum. 1988, 17, 1-21.

25. Maruyama, S; Anderson, L. R.; Smalley, R. E. Rev. Sci. Instrum. 1990, 61, 3686-3693.

26. Alford, I. M.; Weiss, F. D.; Laaksonen, R. T; Smalley, R. E. J. Phys. Chem. 1986, 90, 4480-4482.

27. Henry, K. D.; McLafferty, F. W. Org. Mass. Spectrom. 1990, $25,490-492$.

28. Henry, K. D.; Williams, E. R.; Wang, B. H.; McLafferty, F. W.; 
Shabanowitz, J.; Hunt, D. W. Proc. Natl. Acad. Sci. USA 1989, 86, 9075-9078.

29. Henry, K. D.; Quinn, J. P.; McLafferty, F. W. I. Am. Chem. Sac. 1991, 113, 5447-5449.

30. Hotstadler, S. A.; Laude, D. A. Jr. Anal. Chem. 1992, 64, 569-572.
31. Hofstadler, S. A.; Laude, D. A. Jr. I. Am. Soc. Mass Spectrom. $1992,3,615-623$.

32. G. Scholes, Ed. Atomic and Molecular Beam Methods, Vol. 1; Oxford University Press: New York, 1988; p. 15.

33. Beu, S. C.; Hendrickson, C. L.; Vartanian, V. H.; Laude, D. A Jr. Int. J. Mass Spec. Ion Processes 1992, 113, 59-79. 